\title{
Pharmacogenomics in the treatment of mood disorders: Strategies and Opportunities for personalized psychiatry
}

\author{
Azmeraw T. Amare $^{1} \cdot$ Klaus Oliver Schubert ${ }^{1,2} \cdot$ Bernhard T. Baune $^{1}$
}

Received: 20 July 2017 / Accepted: 11 August 2017 /Published online: 5 September 2017

(C) European Association for Predictive, Preventive and Personalised Medicine (EPMA) 2017

\begin{abstract}
Personalized medicine (personalized psychiatry in a specific setting) is a new model towards individualized care, in which knowledge from genomics and other omic pillars (microbiome, epigenomes, proteome, and metabolome) will be combined with clinical data to guide efforts to new drug development and targeted prescription of the existing treatment options. In this review, we summarize pharmacogenomic studies in mood disorders that may lay the foundation towards personalized psychiatry. In addition, we have discussed the possible strategies to integrate data from omic pillars as a future path to personalized psychiatry. So far, the progress of uncovering single nucleotide polymorphisms (SNPs) underpinning treatment efficacy in mood disorders (e.g., SNPs associated with selective serotonin re-uptake inhibitors or lithium treatment response in patients with bipolar disorder and major depressive disorder) are encouraging, but not adequate. Genetic studies have pointed to a number of SNPs located at candidate genes that possibly influence response to; (a) antidepressants $C O M T$, HTR2A, HTR1A, CNR1, SLC6A4, NPY, MAOA, IL1B, GRIK4, $B D N F$, GNB3, FKBP5, CYP2D6, CYP2C19, and ABCB1 and (b) mood stabilizers (lithium) 5-HTT, TPH, DRD1, FYN, INPP1, CREB1, BDNF, GSK3 $\beta$, ARNTL, TIM, DPB, NR3C1, $B C R, X B P 1$, and $C A C N G 2$. We suggest three alternative and complementary strategies to implement knowledge gained from pharmacogenomic studies. The first strategy can be to implement diagnostic, therapeutic, or prognostic genetic testing based
\end{abstract}

Bernhard T. Baune

Bernhard.Baune@adelaide.edu.au

1 Discipline of Psychiatry, School of Medicine, University of Adelaide, North Terrace, Adelaide, SA 5005, Australia

2 Northern Adelaide Local Health Network, Mental Health Services, Adelaide, SA, Australia on candidate genes or gene products. The second alternative is an integrative analysis (systems genomics approach) to combine omics data obtained from the different pillars of omics investigation, including genomics, epigenomes, proteomics, metabolomics and microbiomes. The main goal of system genomics is an identification and understanding of biological pathways, networks, and modules underlying drug-response. The third strategy aims to the development of multivariable diagnostic or prognostic algorithms (tools) combining individual's genomic information (polygenic score) with other predictors (e.g., omics pillars, neuroimaging, and clinical characteristics) to finally predict therapeutic outcomes. An integration of molecular science with that of traditional clinical practice is the way forward to drug discoveries and novel therapeutic approaches and to characterize psychiatric disorders leading to a better predictive, preventive, and personalized medicine (PPPM) in psychiatry. With future advances in the omics technology and methodological developments for data integration, the goal of PPPM in psychiatry is promising.

Keywords Personalized psychiatry $\cdot$ Lithium · SSRIs · Pharmacogenomics · Depression · Bipolar disorder . Predictive preventive and personalized medicine (PPPM)

\section{Introduction}

Mood disorders in psychiatry encompass a bipolar disorder (BPD), which is characterized by recurrent alternating episodes of elevated mood (mania) and depression, and a major depressive disorder (MDD), which is defined by symptoms associated with pervasively low mood. The clinical diagnosis of MDD is based on the presence of either depressed mood or loss of interest and pleasure (anhedonia) for at least 2 weeks, and requires additional symptoms such as weight loss, 
changes in appetite, insomnia or hypersomnia, psychomotor agitation or retardation, loss of energy or fatigue, feelings of worthlessness, poor concentration, and suicidal thoughts [5]. Both MDD and BPD are among the most disabling mental health disorders worldwide $[34,41,85,120]$ with a lifetime prevalence of $\sim 12$ [4] and $1 \%$ [80], respectively. Based on the 2013 global burden of diseases study by the Institute for Health Metrics and Evaluation at the University of Washington, MDD alone caused an estimated 61.6 million years of life lived with disability worldwide, accounting for $8.1 \%$ of the total years lived with disability (YLDs) and for $2.5 \%$ of the total disability-adjusted life years [33, 41, 85]. BPD contributed an additional 9.9 million years of life lived with disability [34].

The causes of mood disorders are complex and involve the interplay between genetic predisposition and non-genetic biological, psychological, and social factors. Both MDD and BPD are highly heritable, and genetic factors contribute 31$42 \%$ of the disease risk in MDD [109] and 59-85\% in BPD $[71,77]$. Moreover, patients with MDD and BPD often show overlapping clinical features [25]. It is estimated that about $47 \%$ of the genetic risk factors are shared between MDD and BPD [21] and a shared genetic risk was revealed between mood disorders and other psychiatric and medical morbidities [3, 21, 22]. Environmental risk factors such as childhood abuse (physical, sexual, or psychological) are also frequently reported to be associated with both disorders [1].

Candidate gene and genome-wide association studies (GWASs) have identified a list of candidate genes for MDD $[20,24,35,59]$ and BPD [55, 84, 92, 106]. To date, GWAS approaches have identified 18 loci contributing to the risk of MDD [20, 24, 59] and over 8 loci associated with BPD [55, $63,84,92,106]$, including shared genetic loci located in the CACNA1C, CACNB2, AS3MT, ITIH3, and CCDC68 genes [22]. An extension of the GWAS approaches such as polygenic score analysis [87], bivariate restricted maximum likelihood (REML) in genome-wide complex trait analysis (GCTA) [21] and linkage disequilibrium (LD) score regression have strongly suggested that mood disorders are polygenic in nature, in that multiple genetic variants interacting with environmental factors contribute to the development of the diseases [87].

\section{Pharmacogenomics in the treatment of $\operatorname{mood}$ disorders}

After the successful completion of the Human Genome Project, there was an expectation that new genetic discoveries would rapidly and fundamentally improve medical care. For example, it was hoped that pharmacological treatment response and treatment-associated side effects would become more predictable from patients' genetic signatures. However, progress to date has been much slower than initially expected. Part of this delay relates to the fact that an individual's response to pharmacotherapy is multifactorial and involves multiple genes that, in turn, interact with numerous environmental factors [36]. This is a challenge, especially in the pharmacogenomic studies of psychiatric disorders, where underlying traits are extraordinarily complex and heterogeneous. Moreover, it is unclear whether the existing knowledge from pharmacogenomics has yet the necessary and sufficient information for clinical tests and applications. Nevertheless, knowledge of pharmacogenomics is continuing to expand and in the future, it may be possible to integrate genomic data with other biological and clinical information to support decision-making in psychiatric care.

In this review, we discuss recent discoveries in the pharmacogenomics of mood disorders, mainly focusing on treatment response to selective serotonin reuptake inhibitors (SSRIs) in MDD and lithium treatment response in BPD. We also discuss some methodological strategies to integrate genetic evidence into clinical care, as well as future directions towards personalized psychiatry.

\section{Key concepts, definitions, and principles of pharmacogenomics}

Pharmacogenomics focuses on the study of the whole genome genetic variation for its effect on pharmacological treatment outcomes such as therapeutic efficacy or medication side effects. The human genome is composed of roughly 3.1 billion nucleotide bases. The 1000 Genomes Project recently sequenced the genomes of 2504 individuals representing over 26 populations groups and reported a total of over 88 million genetic variants, of which 84.7 million are single nucleotide polymorphisms (SNPs), 3.6 million are short insertions/ deletions (indels), and the remaining 60,000 are structural variants [114]. Our genome has around 30,000 genes and every individual inherits two copies of most genes, one from each parent.

\section{Single nucleotide polymorphisms}

Although the DNA of two individuals is roughly $99 \%$ identical, over 84 million genetic variations occur at the nucleotides level across the human genome [114]. Genetic variants that are found in more than $1 \%$ of the population are called polymorphisms. The most abundant type of genetic polymorphisms, which are found in more than 5\% of the human population are called common SNPs. It is recognized that roughly $54 \%$ of the SNPs are located within the coding region of genes that determine the structure of the gene product (protein) [49]. Thus, a sequence variation within these regions may result in alterations in the encoded protein, which in turn may have an effect on phenotypes (e.g., treatment response). Pharmacogenomics studies how a person's genetic makeup at the nucleotide level influences their reaction to medications. 
It also investigates how genetic variations interact with environmental determinants (microbiome, diet, age, lifestyle, and state of physical health) to influence an individual's response to drugs.

\section{Objectives of pharmacogenomics}

In the context of psychiatry, the main aim of pharmacogenomics is the identification of genes associated with treatment response to psycho-pharmacotherapeutic agents, with an ultimate goal of implementing this information to improve treatment outcomes.

The current psychiatric assessment, clinical decisionmaking and treatment choice is primarily dependent on the clinical experience and professional judgment of psychiatrists, and no known biological marker is yet available to perform either a diagnostic or a prognostic test. The treatment response of patients with mood disorders treated by the current approaches of psycho-pharmacotherapy vary widely between individual patients and is unsatisfactory in many cases [10, 52, 56]. For instance, in patients with MDD, the treatment efficacy of selective serotonin reuptake inhibitors (SSRIs), the most commonly used first-line pharmacological agent [51] varies between 48 and 64\% [10, 52] and reported remission rates are as low as $23.5 \%$ [10, 89]. Similarly, patients treated with lithium in BPD are only partially responsive and more than a quarter of them have no clinical response at all [56]. Thus, an application of findings from pharmacogenomics in mood disorders may help to adjust pharmacotherapy to improve efficacy and to reduce the risk of side effects. The key goals of pharmacogenomics include:

Improving patient care and safety One of the objectives of pharmacogenomics and genetic testing is to identify patients who are likely to respond to treatment and/or experience adverse drug reactions or side effects. Genetic testing may highlight to psychiatrists which patients need particularly careful monitoring and could guide the switching of medications as appropriate.

Improving health care costs and efficiency The resources, including drugs and human resources that are invested in the patient's care, can be optimized by prescribing the right drug for the right patient at the right time.

Individualized adjustment and selection of drugs An understanding of differences in the genetic makeup of patients may help to select the right drug for the "right" patient and allow adjustment of drug dosage according to the likelihood that they will get a favorable response with a lower risk of side effects. Pharmacogenomic agents that show a proven efficacy and safety with a minimal cost as evidenced by clinical trials can be considered as the right drugs. In recent years, pharmacogenomic discoveries and related tests allowed drug selection to be more individualized. This included dosage adjustment, so that maximum efficacy could be achieved with minimal side effects as opposed to the traditional "one size fits all" model of drug selection, which is less useful.

\section{Current state of pharmacogenomic studies in mood disorders}

In the following section, we summarize some of the recent findings from larger-scale pharmacogenomic studies in mood disorders, namely on the genomics of SSRIs treatment response in MDD and of lithium treatment in BPD.

\section{SSRIs treatment for major depressive disorder}

Studies using twin, candidate gene, and genome-wide association study (GWAS) designs suggest that antidepressant treatment response is substantially influenced by genetic factors [6, 7, 8-10, 26, 27, 54, 113]. Although quantitative genetic studies estimated up to $42 \%$ of the individual variation that in antidepressants (e.g. SSRIs) treatment response accounts for genetic factors, progress in uncovering the particular genetic polymorphisms has been slow [112]. As described in Table 1, candidate gene studies have pointed to a number of genes and SNPs that may influence antidepressant treatment outcomes $[6,7,8-10,26,27,54]$, including polymorphisms within the COMT [8, 30], HTR2A [54, 86, 121], HTR1A [26], CNR1 [26], SLC6A4 [9], NPY [6], MAOA [27, 30], IL1B [7, 30], GRIK4 [64], BDNF [30, 86], GNB3 [30], FKBP5 [86], CYP2D6 [31, 121], CYP2C19 [31, 121], and ABCB1 [12, 86] genes (Table 1). For example, a negative influence of a higher activity COMT $158 \mathrm{val} / \mathrm{val}$ genotype on antidepressant treatment response was shown []. In the Sequenced Treatment Alternatives to Relieve Depression (STAR*D) Study, MDD patients who were homozygous for the A allele at rs7997013 within the HTR2A gene demonstrated an $18 \%$ better response to citalopram treatment compared to those homozygous for the G allele [78].

Unfortunately, GWAS have been underpowered to conclusively discover the genetic polymorphisms involved in antidepressants treatment outcomes. To date, several efforts in GWASs of antidepressants reported only suggestive genetic regions and the findings were scarcely replicated in the subsequent studies. The first GWAS involving a total of 339 depressed patents from the Munich Antidepressant Response Signature (MARS) project and a replication analysis using data from the psychiatric hospital of the Ludwig Maximilians University (LMU, $n=361$ ) and STAR*D $(n=832)$ reported two suggestive SNPs rs6989467 in the CDH17 gene associated with partial response 
Table 1 An overview of genetic variants and candidate genes associated with antidepressants treatment response in patients with MDD

\begin{tabular}{lll} 
Candidate genes Coded proteins $\quad$ Pharmacogenomics of antidepressants (description) \\
\hline
\end{tabular}

ILIB

Interleukin-1 beta

\section{FKBP5}

FK506 binding protein 5

CNR1

Cannabinoid receptor 1

\section{$N P Y$}

Neuropeptide Y

$A B C B 1$

ATP-binding cassette subfamily B member 1

\author{
$B D N F$ \\ Brain-derived neurotrophic \\ factor
}

\section{GRIK4}

Glutamate ionotropic receptor kainate-type subunit 4

GNB3

$\mathrm{G}$ protein subunit beta 3

\section{HTR1A}

5-hydroxytryptamine receptor $1 \mathrm{~A}$

\section{HTR2A}

5-hydroxytryptamine receptor

$$
2 \mathrm{~A}
$$

\section{SLC6A4}

Solute carrier family 6 member 4
$I L 1 B$ is one of eight other interleukin-1 family genes that encodes interleukin-1 cytokine proteins. These and other related proteins produced by activated macrophages are important mediators of inflammatory response throughout the body, including in the central nervous system (CNS) [121]

Encodes a protein that is a member of the immunophilin family proteins involved in immune regulation and related cellular processes. This protein binds to the immunosuppressant proteins such as FK506 and rapamycin [121]

Encodes cannabinoid receptor-1, one of the two receptors for cannabinoid - psychoactive ingredients of marijuana [121]

Encodes a neuropeptide that is widely expressed in the CNS and is involved in several physiological processes, including stress response and circadian rhythms [121]

The protein encoded by the $A B C B 1$ gene is a member of ATP-binding cassette $(\mathrm{ABC})$ transporters - superfamily proteins involved in multidrug resistance. $\mathrm{ABC}$ proteins transport molecules across extra- and intra-cellular membranes and functions as a transporter in the blood-brain barrier [121]

Encodes a member of the nerve growth factor family of proteins that promote neuronal survival in the adult brain. $B D N F$ is involved in stress response and in the biology of mood disorders [121]

Encodes a glutamate-gated ionic channel family protein that collectively functions as excitatory neurotransmitters in the CNS [121]

Encodes a beta subunit of the $G$ protein beta family of $G$ proteins (guanine nucleotide-binding proteins) that help to integrate signals between receptor and effector proteins [121]

Encodes a receptor for serotonin that belongs to the

5-hydroxytryptamine receptor subfamily [129].

Serotonin is a neurotransmitter with several roles in the brain and body, and it is assumed to regulate feelings of happiness and well-being [121]

Encodes one of the receptors for serotonin

Encodes a membrane protein that transports serotonin from synaptic spaces into presynaptic neurons, and terminates the action of serotonin and recycles it [121]
Single nucleotide polymorphisms (SNPs) rs16944 and rs116343 in the $I L 1 B$ gene and their interaction with childhood maltreatment influences the effect of antidepressants in patients with MDD [25] and increase the risk of non-remission after antidepressant treatment in patients with MDD [8]

A genetic variant rs1360780 in the FKBP5 gene predicts remission to antidepressant treatment [71] and SNP rs352428 might influence SSRIs treatment outcomes in MDD patients [44]

The CNRI gene polymorphisms rs 806368 and rs 806371 have shown a significant effect on the clinical response to SSRI (citalopram) [114]. An SNP rs1049353 may confer an increased risk to antidepressants treatment resistance [38]

$N P Y$ gene variation rs 16147 was associated with a slow response and remission to antidepressant treatment [37]

In patients with MDD, $A B C B 1$ polymorphisms (rs1045642, rs2032582, and rs1128503) were associated with adjustment to antidepressant dosages to achieve remission [146]. Genotyping for rs2032583 and rs2235015 (TT/GG) may be used for clinical application to optimize antidepressant treatment [19]. Antidepressant treatment outcome was significantly associated with rs203258, rs2235015 [17], rs2032588 [13], and C3435T [83] polymorphisms. Moreover, a significant association was found between SSRIs-related adverse drug effects and SNPs rs2032583 and rs2235040 [33]

Genetic polymorphisms BDNF G196A [168] and rs908867 [61] did influence antidepressant treatment outcome. For example, $B D N F$ G196A polymorphism in part determined the antidepressant effect of both milnacipran and fluvoxamine [168], and the $B D N F$ Val66Met was associated with SSRI treatment resistance [] and may be connected with lithium prophylaxis [137]

The GRIK4 SNPs rs12800734 [72] and rs 1954787 showed a strong association with antidepressant treatment outcome (remission and/or response) [85]

GNB3 C825T polymorphism influences the efficacy of antidepressants in the treatment of MDD [64, 88, 89, $101,166]$

Patients with BPD who carries a 5-HT1A*C/C genotype showed a better response to an antidepressant (fluvoxamine) [140]. SNPs rs 10042486, rs1364043, and rs6295 within the HTR1A gene were significantly associated with antidepressant response [84]

Polymorphisms in 5HT-2A receptor rs17288723 [72], rs7997012 [102, 103], rs9534505 [93], rs6311, rs6313, rs7997012, and rs1928040 [91, 102] were significantly associated with therapeutic response or remission to antidepressants [102]

SNPs within SLC6A4 were associated with antidepressant response [42, 97, 128, 169], 5-HTTLPR pre-treatment genotyping might help to predict treatment remission [125]. Patients with 5-HTTLPR L/L or STin2 12/12 genotype experienced better clinical response to SSRI 
Table 1 (continued)

Candidate genes Coded proteins Pharmacogenomics of antidepressants (description)

\begin{tabular}{|c|c|c|}
\hline & & $\begin{array}{l}\text { treatment [113]. A high-affinity antidepressant-binding } \\
\text { site was found within the serotonin transporter (hSERT) } \\
\text { protein [132]. SNP rs } 8076005 \text { [108] may be a modulator } \\
\text { of antidepressant response. Moreover, the GNB3, } \\
H T R 2 A \text {, and } S L C 6 A 4 \text { genes may act in an interactive } \\
\text { manner to influence antidepressant treatment outcome } \\
{[99]}\end{array}$ \\
\hline $\begin{array}{l}\text { COMT } \\
\text { Catechol-O-methyltransferase }\end{array}$ & $\begin{array}{l}\text { Encodes the COMT enzyme protein that breakdowns } \\
\text { neurotransmitters, such as dopamine, epinephrine, and } \\
\text { norepinephrine. This enzyme might be involved in the } \\
\text { metabolism of drugs [121] }\end{array}$ & $\begin{array}{l}\text { The Val }(108 / 158) \text { Met variation of the COMT gene is } \\
\text { among the most studied polymorphisms associated with } \\
\text { response to antidepressants treatment }[10,13,151] \text { or } \\
\text { electroconvulsive therapy [42] in patients with MDD }\end{array}$ \\
\hline $\begin{array}{l}\text { CYP2C19 } \\
\text { Cytochrome P450 (CYP) } \\
\text { family } 2 \text { subfamily C } \\
\text { member } 19\end{array}$ & $\begin{array}{l}\text { Encodes a member of the CYP superfamily of enzymes } \\
\text { (monooxygenases) - known to metabolize several } \\
\text { drugs, including psychiatric drugs. The CYP enzymes } \\
\text { together with the permeability glycoproteins (PGP) play } \\
\text { a role in eliminating drugs from the brain and body [121] }\end{array}$ & $\begin{array}{l}\text { Genetic variants within this gene are associated with } \\
\text { variable ability to metabolize drugs- genotyping for } \\
\text { these variants may help to classify individuals as poor or } \\
\text { extensive drug metabolizers. CYP } 2 \text { C19 contributes to } \\
\text { the clearance of many antidepressants [139] and an } \\
\text { amino acid residue } 72 \text { plays a key role in the metabolism } \\
\text { of antidepressants by limiting the binding affinities of } \\
\text { CYP2C9 [6] }\end{array}$ \\
\hline $\begin{array}{l}\text { CYP2D6 } \\
\text { Cytochrome P450 family } 2 \\
\quad \text { subfamily D member } 6\end{array}$ & $\begin{array}{l}\text { It is a highly polymorphic gene that encodes an enzyme in } \\
\text { the CYPsuperfamily [121] }\end{array}$ & $\begin{array}{l}\text { Polymorphisms in the CYP2D6 gene may influence the } \\
\text { metabolism and response of antidepressant response [16, } \\
67,90] \text {. Similar to other CYP enzymes, CYP2D6 may } \\
\text { result in a different ability to metabolize drugs (poor or } \\
\text { ultrarapid) }\end{array}$ \\
\hline $\begin{array}{l}\text { MAOA } \\
\text { Monoamine oxidase A }\end{array}$ & $\begin{array}{l}\text { It is one of two genes that encode mitochondrial enzymes, } \\
\text { which catalyzes the degradation of amines (dopamine, } \\
\text { norepinephrine, and serotonin) }\end{array}$ & $\begin{array}{l}\text { Genetic variants of the } M A O A \text { gene may influence } \\
\text { antidepressant treatment response in patients with MDD } \\
{[40,170]}\end{array}$ \\
\hline
\end{tabular}

\section{COMT}

Catechol-O-methyltransferase

CYP2C19

ytochrome P450 (CYP)

family 2 subfamily $\mathrm{C}$ member 19

CYP2D6

Cytochrome P450 family 2

is a highly polymorphic gene that encodes an enzyme in the CYPsuperfamily [121] treatment [113]. A high-affinity antidepressant-binding of antidepressant response. Moreover, the GNB3,

HTR2A, and SLC6A4 genes may act in an interactive manner to influence antidepressant treatment outcome [99]

The Val (108/158) Met variation of the COMT gene is among the most studied polymorphisms associated with response to antidepressants treatment $[10,13,151]$ or electroconvulsive therapy [42] in patients with MDD variable ability to metabolize drugs- genotyping for these variants may help to classify individuals as poor or extensive drug metabolizers. CYP2C19 contributes to clearance of many antidepressants [139] and an of antidepressants by limiting the binding affinities of result in a different ability to metabolize drugs (poor or

antidepressant treatment response in patients with MDD $\left(p=7.6 \times 10^{-7}\right)$ and $\mathrm{rs} 1502174\left(p=8.5 \times 10^{-5}\right)$ in the $E P H B 1$ gene associated with response and remission to antidepressants [62].

The second GWAS on treatment response to the SSRI antidepressant citalopram studied 883 treatment responders and 608 non-responders derived from STAR*D in USA [38]. This study reported three SNPs rs6966038 in the $U B E 3 C$ gene $\left(p=4.65 \times 10^{-7}\right)$, rs6127921 in the BMP7 gene $\left(p=3.45 \times 10^{-6}\right)$, and $\mathrm{rs} 809736$ in the RORA gene $\left(p=8.19 \times 10^{-6}\right)$ that showed suggestive association with treatment response and remission [38]. A similar GWAS on the patterns of treatment response using the STAR $* D$ subjects having sustained ( $n=869)$ versus unsustained $(n=247)$ patterns and a replication attempt at the Genome-Based Therapeutic Drugs for Depression (GENDEP, $n=585$ ) found that the strongest suggestive association was detected at rs10492002 $\left(p=4.5 \times 10^{-6}\right)$ in the ACSS3 gene [58]. A GWAS on susceptibility to antidepressant side effects on STAR*D $(n=1439)$ identified ten linked SNPs in SACMIL gene $\left(p=4.98 \times 10^{-7}\right)$ associated with bupropion's side effects on sexual dysfunction [18]. Similarly, two SNPs rs17135437 $\left(p=3.27 \times 10^{-8}\right)$ within EMID2 gene and intergenic SNP rs16965962 $\left(p=3.22 \times 10^{-7}\right)$ exhibited positive association with the effects of citalopram on vision/hearing [2].
The third GWAS on antidepressant efficacy using patients treated with escitalopram $(n=394)$ and nortriptyline $(n=312)$ from the GENDEP study revealed rs 2500535 $\left(p=3.56 \times 10^{-8}\right)$ in the UST gene associated with nortriptyline response and the rs1126757 $\left(p=2.83 \times 10^{-6}\right)$ in the $I L 11$ gene associated with citalopram response [116].

A further meta-analysis of the three GWAS on antidepressant treatment response from MARS, STAR*D, and GENDEP, which included 2256 participants was also unable to detect SNPs at a genome-wide level of significance [117].

The fourth GWAS on response to serotonergic and noradrenergic antidepressants $(n=1790)$ from the Novel Methods leading to New Medications in Depression and Schizophrenia (NEWMEDS) consortium found no SNP-associated with antidepressant response, even after a meta-analysis of the results from NEWMEDS and STAR*D $(n=2897)$ [113].

The fifth most recent pharmacogenomics study on SSRIs response reported by the International SSRIs Pharmacogenomics Consortium (ISPC) was also unsuccessful to discover genetic variants associated with SSRIs response and remission in MDD patients after 4 weeks of treatment [10]. Similarly, polygenic approaches that tried to elucidate the overall genome-wide genetic influences on antidepressant treatment response in MDD have failed to predict antidepressant efficacy in 
$\operatorname{GENDEP}(n=736)$ or the $\operatorname{STAR} * \mathrm{D}(n=1409)$ studies $[37,53]$. One possible explanation for the difficulty in uncovering genetic variants associated with SSRIs treatment response is that treatment response is clouded by the clinical heterogeneity of MDD as a DSM-defined disorder. A second possible explanation is that SSRIs treatment response likely represents a complex, highly polygenic trait, influenced by a large number of genes with small effects and interacting environmental factors. Increasing the sample size for the current pharmacogenomics studies seems to be the only available option to be successful in this process, as previously been observed in MDD GWAS [59]. Genetic variants associated with MDD were undetectable for smaller sample sizes $(<200,000)$ [59]. The identification of additional variants could be made easier through further advances in sequencing technologies, improved analysis methods and a more homogeneous definition of clinical (sub-) phenotypes.

\section{Lithium treatment for bipolar disorder}

Lithium is the first-line mood stabilizer introduced by the Australian psychiatrist John Cade in 1949 [14]. Since then, it has been used as a first-line treatment of BPD and as prophylaxis to prevent its recurrence [99]. The rate of lithium treatment response [105] and prophylactic efficacy [39] in BPD is relatively high compared to placebo. Yet, data has shown that a substantial proportion of patients hardly achieve acceptable levels of lithium response with a significant interindividual variation between treatment responders and nonresponders [56]. While clinical studies report a combination of demographic and clinical characteristics as potential predictors of treatment response in mood disorder patients [66], genetic factors are also highly involved in lithium treatment response with a potential interplay between genetic and environmental factors $[47,56]$.

Pharmacogenomic studies that aimed to identify genetic variants associated with lithium treatment responses, efficacy, tolerability, and safety in patients with BPD have identified novel SNPs located in protein coding genes. Candidate gene studies have reported several polymorphisms associated with lithium treatment response located in the 5-HTT [101, 102], TPH [100], DRD1 [97], FYN [111], INPP1 [108], CREB1 [73], BDNF [98], GSK3 $\beta$ [70], ARNTL [96], TIM [96], DPB [40], NR3C1 [110], BCR [75], XBP1 [74], and CACNG2 [103] genes (see Table 2).

So far, three GWASs have successfully identified SNPs associated with lithium treatment response in patients with BPD. A GWAS by the International Consortium on Lithium Genetics ( $\mathrm{ConLi}^{+} \mathrm{Gen}$ ), incorporating over 2500 bipolar patients from Europe, USA, Asia, and Australia found a single locus of four linked SNPs on chromosome 21 [56]. Another GWAS by the Taiwan Bipolar Consortium involved 294 bipolar patients of Han Chinese descent and identified two SNPs in high linkage disequilibrium, located in the introns of GADL1 gene [16]. The third GWAS that involved 3874 patients with BPD using subjectively (self-reported) and objectively defined (clinically documented) lithium response data from Sweden and the UK revealed a SNP within the PLET1 gene associated with lithium-responsive patients in BPD []. However, in-depth follow-up functional characterization is required before these associations can be harnessed to improve the understanding of the molecular mechanisms underlying the therapeutic effects of lithium. Findings to date are inconsistent across studies and are far from being helpful in the everyday patient care [56].

It is also interesting to note that some of the candidate genes reported in the pharmacogenomic studies of mood disorders have overlapping effects in response to antidepressants in MDD and lithium treatment response in BPD. For instance, polymorphisms in the SLC6A4 gene were associated with antidepressant response or remission [28, 68, 76, 81, 88, 91]. Similar SLC6A4 variants have shown an association with lithium treatment outcomes in patients with BPD [75, 102].

\section{Polygenic approach to pharmacogenomics in mood disorders}

Genome-wide association studies have consistently demonstrated that psychiatric disorders are highly polygenic, with a large number of genetic variants with small effect underline the susceptibility to the disorders [46]. A similar polygenicity is expected in drug-response phenotypes of mood disorders [10]. One approach that can assist in translating genomic knowledge into clinical applications is the polygenic score (PGS) method proposed by the International Schizophrenia Consortium [93]. This method quantifies the aggregate effect of genome-wide genetic variants, including those polymorphisms that showed only a marginal association with treatment-associated phenotype. Polygenic modeling has an advantage over commonly used cross-trait methods such as LD score regression method or the cross-trait restricted maximum likelihood (REML) approach [21], in that, it is suitable for the clinical application and epidemiological interpretation. An accurate and successful polygenic model may assist early screening for disease risk, clinical diagnosis, and in the prediction of treatment response and disease prognosis. Thus, polygenic profiling may help to stratify patients by the status of drugs response, then helping to choose or refine treatments. Moreover, PGS might be used as a quantitative measure of genetic load that can be tested for its association with endophenotypic measures, such as plasma level of drugs or halflife. PGS associations can also be translated into odds ratios by comparing the highest versus lowest quartile (decile) groups, which makes clinical interpretations more simplified. Whether these PGSs have the potential to be applied to everyday clinical care depends on their ability to differentiate patients into categories of treatment responders versus non- 
responders or remitters versus non-remitters. More research is required to determine whether this is the case, and future studies should consider related variables such as multi-trait PGSs analysis, or interaction analyses with clinical and environmental factors, i.e., $\mathrm{PGS} \times \mathrm{PGS} \times$ environment interactions.

The success of PGSs is likely to depend on three important factors: (a) the power of the initial GWAS studies, (b) homogeneity of the population, and (c) sample size. It has been shown that polygenic score is likely to be useful when it is population-specific (in a homogeneous population) and when the initial GWAS is successful.

An additional implication of using a PGS is that it provides platforms for understanding convergence in co-morbid diseases and their influence on treatment outcomes. A recent review of GWAS and candidate gene studies provided evidence for a cross-disorder genetic overlap between mood disorders and cardiometabolic abnormalities. This evidence indicated a genetic basis for the impact that medical comorbidities have on treatment outcomes in mood disorders [3]. Polygenic analysis can be applied in such cases to estimate the extent of genetic overlap as well as to identify polygenic predictors [3].

\section{Challenges of pharmacogenomics studies in mood disorders}

Pharmacogenomics studies in mood disorders have specific challenges in addition to those common to other genetic studies. Generally, accumulating an adequate number of patients, precisely defining drug-response phenotypes and addressing analytic issues of large data are the challenges to the successful accomplishment of pharmacogenomic studies. Additional challenges specific to mood disorders include the heterogeneity of the disorders, often overlapping with other medical and psychiatric morbidities, which may result in a heterogeneous treatment outcome (see box 1). Until a more stratified diagnosis and treatment are established, the only way to improve the power of the existing pharmacogenomic studies in mood disorders is increasing the sample size and optimizing the study designs to involve more severe cases. Data from large samples of patients treated with a single treatment option is also very rare, and the dosage of the drugs also vary, further complicating efforts to study the pharmacogenomics of specific drugs. Moreover, patients with mood disorders are often treated with a combination of psychological and pharmacological treatment alternatives, and it is sometimes difficult to rule out whether the patients are benefiting from pharmacological or nonpharmacological treatment, especially when the treatment effect is low. Designing a large pharmacogenomics study is very costly adding clinical trial requirements to the genotyping costs. As the cost of sequencing and genotyping continue to decline, every effort should be made to increase sample sizes of the current projects in order to carry out comprehensive pharmacogenomic studies.

Box 1: Challenges of pharmacogenomics studies in mood disorders
Potential challenges

Establishing a hard-end definition of treatment outcome (response and remission)

Optimizing sample size

Bioinformatics tools for integrative analysis

Replication of findings and moving to clinical application

\section{Description}

Mood disorders are highly heterogeneous and there are no objective criteria to define both the diagnosis of the disorders and treatment outcomes. Assessment tools applied in the diagnosis and treatment follow-up of mood disorders have poor sensitivity and specificity

Underpower is the major bottleneck for success in the pharmacogenomic studies in mood disorders and a global collaboration is required to improve the existing efforts

To provide an evidence-based decision about a patient, a complete evaluation of data obtained from the different pillars of omics investigation, including genomics, epigenomes, proteomics, metabolomics, and microbiomes is required. Advanced bioinformatics tools are required to perform such kind of integrative analysis

Findings from both candidate gene studies and GWASs are hardly replicated and translating the findings is inadequate
Generally, when we plan pharmacogenomic studies in mood disorders, particular care needs to be exercised during (a) choosing the patient, (b) designing the study and estimating the sample size, and (c) planning statistical analysis and replication.

The appropriate sample size to design a pharmacogenomics study in mood disorders depends on the expected effect sizes that genetic variants have on the treatment outcome, as well as on the number of independent tests (number of variants) to be tested for their association with the treatment outcome. Thus, the sample size of a study is dictated by the study design. Candidate gene studies include fewer variants than GWAS; therefore, candidate gene studies can enroll a much smaller number of participants. Nonetheless, GWAS require hundred thousands to millions of individuals to detect the effect of genetic variants with a small effect — odds ratios from 1.1 to 2 [107]. It is unlikely that individual clinical trials in a single country will achieve such a sample size, and therefore, optimizing the design of pharmacogenomic studies require global collaboration. To be 
Table 2 An overview of genetic variants and candidate genes associated with lithium response in patients with bipolar disorder

\begin{tabular}{ll}
\hline Candidate genes & Coded proteins \\
\hline$X B P 1$ & $\begin{array}{l}\text { Encodes a protein that regulates MHC class II genes by } \\
\text { binding to an X-box (a promoter element), and it is in- } \\
\text { volved in endoplasmic reticulum stress response [90] }\end{array}$
\end{tabular}

\section{INPPI \\ Inositol \\ polyphosphate-1-- \\ phosphatase \\ CREB1 \\ cAMP responsive element binding protein 1 \\ GSK3B \\ Glycogen synthase kinase 3 beta \\ $\mathrm{NR} 3 \mathrm{Cl}$ \\ Nuclear receptor subfamily 3 group C member 1}

DRD1

Dopamine receptor D1

\section{FYN}

FYN proto-oncogene, Src family tyrosine kinase

Circadian clock genes

TIMELESS

Timeless circadian clock

PER3

Period circadian clock 3

CLOCK

Circadian locomotor output cycle kaput

ARNTL

Aryl hydrocarbon receptor nuclear translocator-like

TPHI

Tryptophan hydroxylase-1

\section{SLC6A4}

Solute carrier family 6 member 4

CACNG2

Calcium voltage-gated channel auxiliary subunit gamma 2

ASIC2

Acid-sensing ion channel subunit 2

GADL1

Glutamate decarboxylase-like 1
Encodes inositol polyphosphate-1-phosphatase, one of the enzymes involved in phosphatidylinositol signaling pathways [90]

Encodes a transcription factor (a member of binding proteins) that induces transcription of genes in response to hormonal stimulation of the cAMP pathway [90]

Encodes serine-threonine kinase, a protein belonging to the glycogen synthase kinase subfamily that are involved in energy metabolism and neuronal cell development [90]

Encodes a glucocorticoid receptor that binds to glucocorticoid responsive genes to activate their transcription. This protein is involved in inflammatory responses [90]

Encodes the dopamine receptor D1, the most abundant dopamine receptor in the CNS. D1 receptors regulate neuronal growth and development. This protein mediates emotion processing and behavioral response [90]

Encodes a tyrosine kinase oncogene family protein implicated in the control of cell growth [90]

TIMELESS, PER3, CLOCK and ARNTL are members of the Period family of genes mainly involved in the control of circadian pattern. They also control cell survival after damage or stress, locomotor activity, metabolism and behavior [90]

Encodes a member of the aromatic amino acid hydroxylase family protein, involved in the biosynthesis of serotonin [90]

Encodes a membrane protein that transports the neurotransmitter serotonin from synaptic spaces into presynaptic neurons [90]

Encodes a type I transmembrane AMPA receptor regulatory protein (TARP) that regulates the channel gating of the AMPA receptors and mediate fast neurotransmission in excitatory synapses [90]

Encodes a member of the degenerin/epithelial sodium channel superfamily that may play a role in neurotransmission [90]

Encodes a protein that catalyzes the decarboxylation of aspartate, cysteine sulfinic acid, and cysteic acid to beta-alanine, hypotaurine, and taurine, respectively [74, 90]
Pharmacogenomic of mood stabilizers, lithium

(description)

Polymorphisms in XBP1 $(-116 \mathrm{C} / \mathrm{G})$ showed a significant association with prophylactic treatment response to mood stabilizers - valproate [66] and lithium [78] in patients with BPD

A polymorphism in INPPI C973A might be associated with the efficacy of lithium [110]. Significant interactions were also found between lithium response and INPPI SNP rs206472 [14]

Variations in the $C R E B 1$ gene were associated with lithium response [77]

$G S K 3 B$ genetic variants may underlie therapeutic response to lithium $[64,73]$ and patients with TT genotype at rs334558 had a poorer response to lithium treatment [73]

NR3C1 polymorphisms (rs6198, rs6191, rs6196, rs258813, rs33388) have shown a significant association with lithium treatment response in patients with BPD [112]

DRDI gene genotype has been associated with a poorer prophylactic effect of lithium [98]

FYN gene polymorphism rs3730353 was associated with prophylactic response to lithium in bipolar patients [113]

Polymorphism in the circadian clock genes may be associated with lithium treatment and prophylactic response in bipolar illness [97]

TPH variants were associated with the prophylactic efficacy of lithium in mood disorders [101]

Possible association between SLC6A4 variants and lithium treatment outcomes in patients with BPD $[79,103]$

CACNG2 gene polymorphisms (rs2284017, rs2284018, rs5750285) were associated with response to lithium treatment [104]

ASIC2 (ACCN1) gene is a potential candidate gene for response to lithium treatment in bipolar patients [109]

Two linked SNPs, rs17026688 and rs17026651 mapped to GADL1 gene showed associations with lithium response in patients with BPD [17] successful, collaborations among geneticists, methodologists, pharmacologists, clinicians, and pharmaceutical companies should be encouraged and cross-consortia partnerships may be required. Some examples of global collaboration efforts in the 
pharmacogenomics studies of mood disorders are described below. They can be considered as future opportunities for pharmacogenomic studies in mood disorders.

\section{International collaboration as opportunity to pharmacogenomic studies in mood disorders}

The international collaboration between scientists in academia, as well as with the pharmaceutical industries provides excellent opportunities for pharmacogenomic studies in mood disorders. In the following section, we introduce some of these initiatives.

\section{Collaborations to study the pharmacogenomics of antidepressants}

Clinical trials and international consortia with available GWAS data in the field of antidepressant response include, the International SSRIs Pharmacogenomics Consortium (ISPC) study [10], Depression and Sequence of Treatment (DAST) Study, the NEWMEDS consortium [37, 57, 113], the Pharmacogenomics Research Network Antidepressant Medication Pharmacogenomics (PGRN-AMPS) Study [83], the Sequenced Treatment Alternatives to Relieve Depression (STAR*D) Study [95], GENDEP project, and the MARS project $[37,50$,$] .$

\section{International SSRI Pharmacogenomics Consortium study} The International SSRI Pharmacogenomics Consortium (ISPC) is an international collaboration of experts established with the aim to discover genetic variants that are responsible for SSRIs treatment outcomes in MDD patients. The first genome-wide association study by this group was published in 2015 [10]. This group has data on 998 MDD patients collected from different countries including in Europe, USA, and Asia. The genotyping for the ISPC sample was performed at the RIKEN Center for Integrative Medical Sciences (Yokohama, Japan). Demographic and clinical data for the ISPC are available at the Pharmacogenetics and Pharmacogenomics Knowledge Base (PharmGKB; www. pharmgkb.org). The depressive symptom measure in the ISPC data was the 17-item Hamilton Depression Rating Scale. Further details are available elsewhere [10].

Depression and Sequence of Treatment study The Depression and Sequence of Treatment (DAST) study is a prospective naturalistic treatment study of 746 inpatients diagnosed with a major depressive disorder of adult age (>18 years of age) collected at the Department of Psychiatry and Psychotherapy at the University of Münster, Germany [7, $8,37]$. MDD was diagnosed using a structured clinical diagnostic interview (SCID). Treatment response and remission were assessed using the Hamilton Depression Rating Scale
(HAM-D) as the primary treatment outcome measure administered on a weekly basis at least for 6-week duration by trained psychiatrists and psychologists. Patients are of Caucasian ancestry and treatment selection was made by clinicians and included flexible antidepressant dosage and agents for augmenting [7, 8, 37].

Pharmacogenomics Research Network Antidepressant Medication Pharmacogenomics Study The Pharmacogenomics Research Network Antidepressant Medication Pharmacogenomics Study (PGRN-AMPS) is a single-arm clinical trial focused on the response of MDD patients to citalopram or escitalopram over 8 weeks of treatment. The study involved 530 participants with nonpsychotic MDD aged between 18 and 84 years, who were recruited from the inpatient and outpatient practices of the Department of Psychiatry and Psychology at Mayo Clinic in Rochester, Minnesota. Depression severity in this cohort was assessed using the 16-item Quick Inventory of Depressive Symptomatology (QIDS-C16). Details on the PGRN-AMPS can be found elsewhere [83].

Sequenced Treatment Alternatives to Relieve Depression study The Sequenced Treatment Alternatives to Relieve Depression (STAR*D) study is a multilevel clinical trial of outpatients with nonpsychotic MDD funded by the National Institute of Mental Health. The STAR*D initially enrolled 4041 MDD patients aged between 18 and 75 years [95], who received the SSRI citalopram in the first 12 to 14 weeks (level 1). Clinical data for the STAR*D was collected using the QIDS-C16 scale. Data on the covariates such as age, gender, and on the specific SSRI medications were also gathered, and the details can be found elsewhere [10, 32, 83].

The Genome-based Therapeutic Drugs for Depression project The Genome-based Therapeutic Drugs for Depression (GENDEP) project involved 868 Caucasian ancestry patients aged 18 to 75 years and is a 12 -week multicenter partly randomized pharmacogenetics trial with two active treatments (an SSRI-escitalopram and a tricyclic antidepressant-nortriptyline) [117].The GENDEP research project is an integrated project involving scientists from ten countries, including Germany, UK, Ireland, Belgium, Italy, Sweden, Denmark, Poland, Slovenia, and Croatia. Investigators at the UK Medical Research Councils (MRC) Social, Genetic, and Developmental Psychiatry Centre (SGDP) at the Institute of Psychiatry, Kings College London, lead the project. The diagnosis of depression was per the ICD-10/DSM-IV criteria and the primary treatment outcome measure was the Montgomery-Åsberg Depression Rating Scale (MADRS).

Munich Antidepressant Response Signature project The Munich Antidepressant Response Signature (MARS) project 
(http://www.mars-depression.de) is a prospective naturalistic study of 842 inpatients aged 18 to 75 years admitted to hospitals in southern Germany for the treatment of MDD or BPD. Diagnoses are based on DSM-IV criteria and a clinical interview by trained psychiatrists $[37,50$,$] . This project was$ initiated in 2000 by the Max Planck Institute of Psychiatry (MPI-P, Munich, Germany), and longitudinal data were collected from three clinical sites in southern Bavaria (MPI-P, Munich; Bezirkskrankenhaus Augsburg; Klinikum Ingolstadt). All patients are of Caucasian ancestry, and treatment was selected by clinicians and included flexible antidepressants dosage and agents for augmenting. The Hamilton Depression Rating Scale (HAM-D) was the primary treatment outcome measure, which was administered weekly by trained psychiatrists and psychologists $[37,50$,$] .$

The GENetic and clinical Predictors of treatment response in Depression study The GENetic and clinical Predictors Of treatment response in Depression (GENPOD) study was a multicenter RCT conducted in Bristol, Birmingham, and Newcastle, UK, involving 601 patients (men $n=161$, women $n=347$ ) aged $18-74$ years recruited in primary care who had an ICD-10 diagnosis of MDD who were randomized to either a SSRI (citalopram) or a NARI (reboxetine) [115].

Novel Methods Leading to New Medications in Depression and Schizophrenia consortium This is an international consortium of research academic-industry collaboration aimed to find new methods for the development of drugs for schizophrenia and depression. Individual clinical trials currently part of the Novel Methods Leading to New Medications in Depression and Schizophrenia (NEWMEDS) consortium (http://www.newmeds-europe.com) include three studies from academic institutions (GENDEP, $n=868$; GENPOD, $n=601$; and Geneva Outpatient Depression Study (GODS), $n=131$ ) and two studies by the European Federation of Pharmaceutical Industries and Associations (Pfizer, $n=355$, and GlaxoSmithKline (GSK), $n=191$ ). In all, NEWMEDS has enrolled 2146 individuals (941 men and 1205 women) diagnosed with MDD according to the ICD-10 and DSM-IV, with data on the prospective outcome of treatment with norepinephrine reuptake inhibitors (NRIs) or selective serotonin reuptake inhibitors (SSRIs) [37, 57, 113]. These patients underwent 6 to 12 weeks of treatment with either SSRIs (citalopram, escitalopram, sertraline, paroxetine, fluoxetine) or NRIs (reboxetine, nortriptyline) [37, 57, 113].

The Geneva Outpatient Depression Study The GODS study [11] is a partly randomized trial led by researchers at Geneva University Department of Psychiatry, which examined the efficacy of four antidepressants (clomipramine, paroxetine, nefazodone, and venlafaxine) in a cohort of 131 subjects (53 men and 78 women) with MDD patients aged $18-65$ years $[11,113]$.

Pfizer: pharmaceutical company The study by Pfizer involved a total of 355 MDD patients from eight clinical facilitates primarily conducted as double-blind, placebo-controlled trial, with follow-up for 6 to 8 weeks. The patients in the treatment groups had sertraline, fluoxetine or paroxetine [113].

GlaxoSmithKline: pharmaceutical company The samples for the GlaxoSmithKline (GSK) study were derived from two randomized studies conducted from January 2003 to June 2004 in the USA to compare the efficacy of antidepressants and the effect of bupropion and escitalopram on sexual functioning in outpatients with depression $(n=210)[19,113]$.

\section{Collaborations to study the pharmacogenomics of lithium}

The International Consortium on Lithium Genetics The International Consortium on Lithium Genetics (ConLiGen) consortium is the largest gathering in the genetics of lithium to date that aims to identify genetic polymorphisms associated with lithium treatment response in BPD, as well genetic determinants of adverse events that may be resulted from lithium treatment. The ConLi ${ }^{+}$Gen Consortium (www.ConLiGen.org) is an initiative by the National Institute of Mental Health (NIMH) and the International Group for the Study of Lithium-Treated Patients (IGSLI) (www.IGSLI.org) [99]. The ConLiGen has compiled genetic and clinical data from 3193 patients with BPD who had undergone lithium treatment in Europe, USA, Asia, and Australia [56]. The first GWAS was published in 2016 [56]. ConLiGen has continued to invite researchers around the world, including from developing countries, to join the current efforts of increasing sample size and to adequately represent the patient population.

\section{Strategies and future paths to personalized psychiatry}

The terms "precision," "personalized," and "individualized" medicine are often used interchangeably, and refer to the treatment of patients based on individual characteristics including genomic information [42, 65]. The European Association for Predictive, Preventive and Personalized Medicine (EPMA) promotes this integrative concept of medicine [43-45, 69] and describes the predictive, preventative and personalized medicine (PPPM) as the medicine of the future. PPPM is one of the main strategies in "Horizon 2020" [43, 45, 69]. Horizon 2020 is the biggest European Union Research and Innovation programme with a funding budget of nearly 80 billion euros over 2014 to 2020 .

With pharmacogenomics being one focus in the process towards personalized psychiatry, we suggest three alternative 


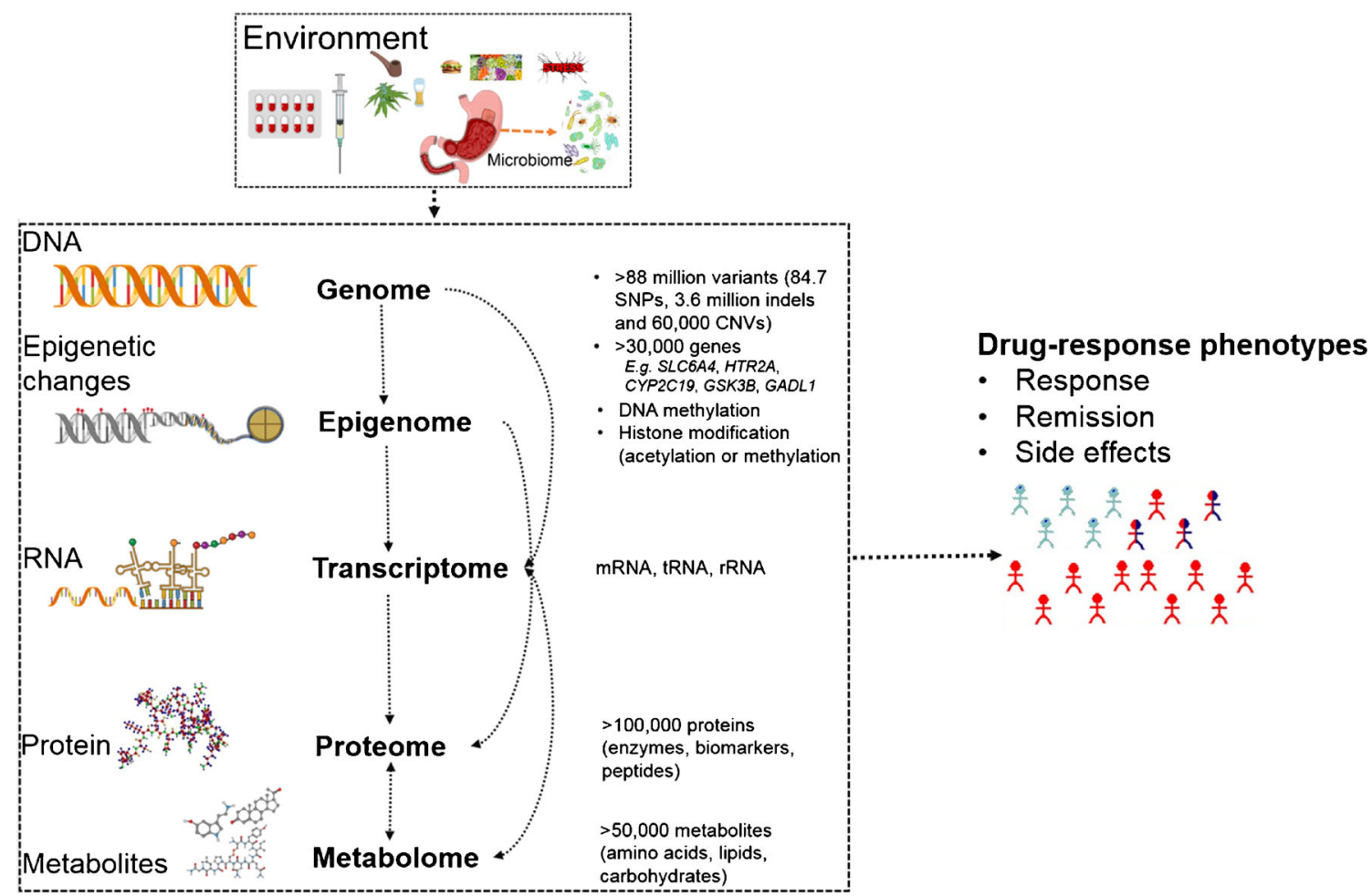

Fig. 1 An overview of the biological data that need to be integrated into the systems genomics approach to investigate pathways in complex traits (e.g., treatment response)

and complementary strategies to implement knowledge generated from genetic studies. The first plan could be to design a therapeutic or diagnostic genetic testing scheme based on candidate genes or gene products - testing their interactions with psychopharmacological drugs and predict treatment outcomes. The second strategy refers to an integrative analysis of -omics data obtained from the different pillars of omics investigation including genomics, epigenomes, proteomics, metabolomics, and microbiomes. Biologists have labeled this procedure as "systems genomics (genetics)" approach. The main goal of systems genomics is an identification and understanding of biological pathways, networks, and modules affected by underlying complex genetic traits. These pathways could be used as drug targets and may guide the efforts to new drug development. The third approach aims to develop multivariable prediction algorithms for diagnostic or prognostic purposes.

\section{Strategy 1: genetic testing based on candidate genes or gene products}

The clinical utility of pharmacogenomics evidence and subsequent genetic testing in psychiatric care is an ongoing process given that studies are generating new evidence. The current approach of genetic testing is based on "targeted genotyping" of genetic variations in genes that are involved in the metabolism of many drugs including psychiatric medications. An example of pharmacogenomic testing in psychiatry is the genotyping for drug-metabolizing enzyme genes, known as cytochromes P450 (CYP) [90, 104, 118]. Variations within the CYP genes are extensive and have long been known to affect the metabolism of several drugs, including antidepressants and lithium. Hence, the CYP genes have been extensively studied in psychiatry. There are now commercially available gene chips to genotype these genetic variations for patients who are receiving antidepressants or mood stabilizers [82]. For example, a commercial pharmacogenetics test (GeneSight) helps to identify individuals who are either poor metabolizers or ultrarapid metabolizers of over 55 neuropsychiatric drugs based on the genotype of the CYP (CYP2D6, CYP2C19, CYP2C9, and CYP1A2) [82, 121]; SLC6A4; $H T R 2 A$; and $A B C B 1$ genes [121].

\section{Strategy 2: integrative analysis-system genomics approach}

System genomics — broadly called systems biology — is a global perspective of understanding the mechanisms underlying complex traits [17, 94]. It involves a range of experimental and statistical modeling techniques aimed to integrate broad data from genomes (DNA), epigenomes, transcriptomes (RNA), proteomes, metabolomes, and their interactions with 
the environment such as with the microbiomes [17, 94] (see Fig. 1). System genomics can be applied in drug-response phenotypes of mood disorders - to identify biological pathways, networks, and modules that may ultimately be used as targets for new drug development. This approach is complementary to the other strategies suggested in our review, but specifically aimed to understand molecular underpinnings of complex traits, rather than the development of diagnostic or prognostic algorithms (tools) for immediate application. The statistical techniques are advanced from a single phenotypesingle data approach (single omics data) to meta-dimensional and multistage analysis (multiple omics approaches). The analysis methods include, among others, expression quantitative trait loci (eQTL), pathway analysis, Bayesian networks, evolutionary computing methods, symbolic regression, and artificial neural networks (ANNs). These data integration techniques can be categorized into two, as a multi-staged analysis and meta-dimensional analysis approach. In a multistaged modeling, the analysis is divided into different steps to test the associations between the different data types and the phenotypes, allowing for only two different scales at a time (i.e., continuous and categorical scales). Conversely, a metadimensional modeling combines multiple data types and all scales simultaneously to identify complex, meta-dimensional models with multiple variables from different data types. Software tools have been developed to implement several techniques of multi-staged, meta-dimensional analysis and/ or a combination of these techniques. Details of these methods are available elsewhere [17, 94].

\section{Strategy 3: development of prognostic models}

While efforts continue to improve the power of the existing GWAS studies, we are also beginning to see the opportunities and challenges in implementing genomic information to personalized psychiatric care. Until all the genetic discoveries are successful and validated with high confidence, the clinical utility of genetic variants requires careful implementation and integration of innovative algorithms that capture the overall variance in a complex trait (e.g., treatment response) accounted by genome-wide genetic variants. In this regard, the polygenic modeling method allows capturing of the contribution of genome-wide SNPs to complex phenotypes, such as pharmacogenomic traits. Clinical translation of polygenic findings may be challenging, but future advances in sequencing technologies and novel statistical genetics analysis methods may improve the predictive power of polygenic scores. In the future, every individual's genotype data may be readily available, and improved algorithms that can combine the genetic effects of every polymorphism including from rare genetic variants may be developed. Until then, the goal should be on the identification of the remaining genetic variants for treatment response and implement at least a semi- powerful polygenic approach. For better use of the current scientific output, prognostic models combining data from biological, clinical, psychosocial, and sociodemographic predictors could be developed and tested.

Prognostic models that are developed based on several patient characteristics [23, 29, 67], including genetic information are a cornerstone of precision medicine. Despite limited available information, structural and functional networks in the brain [29] including neuronal connections [67], microbiome [23], epigenetic mechanisms [79, 119], and changes in gene expression [13] are all associated with drug efficacy and could be considered as additional markers to predict treatment response in a clinically meaningful way. The capacity to combine all potential genetic and non-genetic factors that possibly affect treatment response to psycho-pharmacotherapy determines the future path to personalized psychiatry.

The concept behind the development of prognostic models is to estimate the probability that a patient with a given set of characteristic (predictors) will respond favorably or poorly to the pharmacological treatment. Such models are developed based on the patients' baseline characteristics - likely to be associated with the prognosis of the disorders and/or the efficacy of the drugs (see Fig. 2 and Box 2). These predictors are usually selected based on biological evidence, clinical experience, and findings from the literature. Given these characteristics, prognostic models allow estimation of the probability that a patient will favorably respond or poorly react to the drugs. Once such models are developed, their predictive accuracy should be evaluated before they are applied in clinical practice. This process is known as model validation and it involves calibration assessment (testing the agreement between the observed treatment outcomes and predictions) and discrimination assessment (evaluation of the model's capability to discriminate between, e.g., responders and non-responders). Models are usually validated both internally (for example, using bootstrapping) and externally using data from other patient population not used for model development.

Box 2: Approaches in prognostic model development and validation

1. Step wise model development

As an example, we present the principle statistical procedure to construct a prognostic model for mood disorders.

Treatment outcome: Response to treatment (favorable versus poor)

Predictor variables: Age, sex, genetic predictors (e.g., polygenic score for potential predictors), disease severity and other clinical characteristics of the disease at baseline, including psychosocial variables, and co-morbidities (see Fig. 2)

A prognostic model estimates the patient's probability to respond to treatments given a set of predictor variables at the baseline. The appropriate statistical modeling choices for binary outcomes such as treatment response can be:

(a) A binary logistic regression model (binary outcome): This model takes the forms

Probability of response $=\frac{\exp ^{\text {(patien's 's response score })}}{1+\exp ^{\text {(patient's response score })}}$ 
where

Patient's response score $=$ intercept $+\beta 1$ age $+\beta 2$ sex $+\beta 3$ genetic score $+\beta 4$ illness severity at baseline $+\ldots)$.

The beta's $\left(\beta_{1}\right.$ to $\left.\beta_{n}\right)$ are regression coefficients for each predictor variable.

(b) Survival analysis using Cox proportional hazards model, predicts treatment outcomes (e.g. response) at varying time points. This model has the form

hi $(t)=\operatorname{ho}(t) \times \exp ^{[\text {intercept }+\beta 1 \text { age }+\beta 2 \text { sex }+\beta 3 \text { genetic score }+\beta 4 \text { illness }}$ severity at baseline $+\ldots]$

where hi $(t)$ is the expected hazard (response) for individual $i$ at time $t$, and ho $(t)$ is the baseline hazard and represents the hazard when all of the predictors (or independent variables) are equal to zero.

External validation

Models developed in the above procedure should be externally validated using a new group of patients treated with the same drug to predict treatment outcomes using similar predictors. Regression coefficients estimates (from the model development phase) are used to predict the probability of patients' response in the validation sample. The agreement between the predicted and observed outcomes is assessed, i.e., the model is assessed for its performance, for example, calibration and discrimination.

The use of external cohorts increases the generalizability of the model. In case no external patient sample is not available, a bootstrap validation may be used.

2. Machine learning is a recently evolving method, which often overlaps with the above statistical approaches. Essentially, a machine learning explores the data structure using provided instructions (algorithms) to detect patterns of statistical regularity across the data to make meaningful classifications and build prediction models. Supervised machine learning approaches are often suggested as a powerful tool in medicine. Several methods in machine learning use a multivariate approach to the entire dataset and are able to handle interactions $[60,72]$.

Given the complex nature of the predictors for treatment response in mood disorders, a broader understanding of potential predictors has an advantage to enhance the predictive

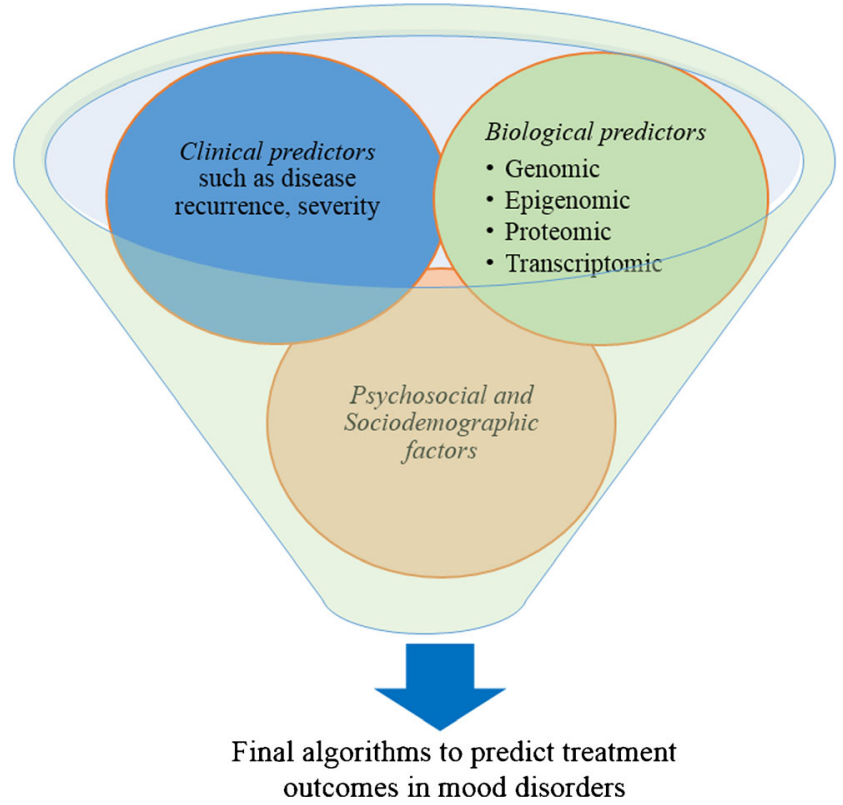

Fig. 2 Overview of how potential predictors can be pooled into a statistical model during the development of algorithms to predict treatment outcomes in mood disorders (e.g., treatment response) capacity of diagnostic or prognostic algorithms (tools). Essentially, predictor variables should be collected in a wider scope including the characteristics that showed weak associations. As shown below, the potential predictor variables in mood disorders can be broadly categorized as biological predictors, clinical characteristics, sociodemographic factors, and psychosocial factors. Although a detailed review of each potential predictor is beyond the scope of this article, the specific biomarkers in each category are usually selected based on biological evidence, clinical experience, and findings from the literature. Once this information is collected, statistical techniques such as machine learning can be applied to produce the final model with the best predictors [61]. During the data mining process, all potential predictors will be pooled and variables that better improve the model will be finally filtered and remained in the model — as measured by for example area under the ROC curve (see Fig. 2). Application of machine learning approaches to predict treatment response has shown promising results to predict treatment outcomes (e.g., in antidepressants) [15]. Analysis of the STAR*D data has identified over 25 variables most predictive of antidepressants treatment outcome with an accuracy ranging between 59.6 and $64.6 \%$ [15]. A similar approach using peripheral gene expression data in major depression identified a panel of 13 genes which predicted citalopram treatment response with a 76-79\% accuracy [48]. These findings are encouraging, but they reported low predictive accuracy indicating that an additional or stronger predictor variable(s) are required. This is the stage at which emerging predictors such as genomic information can help to improve the performance of existing models.

\section{Conclusions and expert recommendations}

In conclusion, a number of pharmacogenomic studies have been conducted to uncover genes associated with treatment response to mood stabilizers and antidepressants. Indeed, the findings from candidate gene and GWAS were encouraging, but not adequate in terms of their power to identify the expected number of genetic variants. Strong international collaboration between scientists in academia and the pharmaceutical industry are important to improve the power of the existing GWAS studies and to achieve the goals of personalized psychiatry.

Findings from pharmacogenomic studies have the potential to improve psychiatric care and many advances are expected in the near future with improvements in the definition of clinical phenotypes, advancement in sequencing technology, and better statistical tools to analyze a broad range of data. The integration of molecular science with that of traditional clinical practice is the way forward to drug discoveries, novel therapeutic approaches, and to characterize the disorders. Suggested strategies to translate the current knowledge into 
clinical practice includes genetic testing, integrative analysis (systems genomics approach), and the development of multivariable diagnostic or prognostic algorithms (tools) to predict therapeutic outcomes. The integration of omics data with clinical variables could lead to a better predictive, preventive and personalized medicine (PPPM) in psychiatry, for example, helping to distinguish patients with favorable response to pharmacological treatment. Omics studies so far were inadequate and had limited power. Further studies should build up from the existing efforts of international collaborations to increase sample size and identify additional biological markers - then data integration and implementation into standard clinical decision-making has a chance to be realized in the future. This would be a major step towards PPPM in psychiatry. While we are still in the early stages of this "revolution", significant scientific innovation gives the field hope to shape the future of psychiatric medicine.

\section{Compliance with ethical standards}

Funding The University of Adelaide, Adelaide Graduate Centre, supported this work. ATA was a recipient of a postgraduate study scholarship, Adelaide Scholarship International (ASI), from the University of Adelaide.

Conflict of interest The authors declare that they have no conflict of interest.

\section{References}

1. Aas M, Henry C, Andreassen OA, et al. The role of childhood trauma in bipolar disorders. Int $\mathrm{J}$ Bipolar Disord. 2016;4:2.

2. Adkins DE, Clark SL, Aberg K, et al. Genome-wide pharmacogenomic study of citalopram-induced side effects in STAR*D. Transl Psychiatry. 2012;2:e129.

3. Amare AT, Schubert KO, Klingler-Hoffmann M, et al. The genetic overlap between mood disorders and cardiometabolic diseases: a systematic review of genome wide and candidate gene studies. Transl Psychiatry. 2017;7:e1007.

4. Andrade L, Caraveo-Anduaga JJ, Berglund P, et al. The epidemiology of major depressive episodes: results from the International Consortium of Psychiatric Epidemiology (ICPE) surveys. Int J Methods Psychiatr Res. 2003;12:3-21.

5. American Psychiatric Association, DSM-5 Task Force. Diagnostic and statistical manual of mental disorders: DSM-5. Arlington: American Psychiatric Association; 2013.

6. Baffa A, Hohoff C, Baune BT, et al. Norepinephrine and serotonin transporter genes: impact on treatment response in depression. Neuropsychobiology. 2010;62:121-31.

7. Baune BT, Dannlowski U, Domschke K, et al. The interleukin 1 beta (IL1B) gene is associated with failure to achieve remission and impaired emotion processing in major depression. Biol Psychiatry. 2010;67:543-9.

8. Baune BT, Hohoff C, Berger K, et al. Association of the COMT val158met variant with antidepressant treatment response in major depression. Neuropsychopharmacology. 2008;33:924-32.
9. Baune BT, Hohoff C, Mortensen LS, et al. Serotonin transporter polymorphism (5-HTTLPR) association with melancholic depression: a female specific effect? Depress Anxiety. 2008;25:920-5.

10. Biernacka JM, Sangkuhl K, Jenkins G, et al. The International SSRI Pharmacogenomics Consortium (ISPC): a genome-wide association study of antidepressant treatment response. Transl Psychiatry. 2015;5:e553.

11. Bondolfi G, Aubry JM, Golaz J, et al. A stepwise drug treatment algorithm to obtain complete remission in depression: a Geneva study. Swiss Med Wkly. 2006;136:78-85.

12. Breitenstein B, Scheuer S, Pfister H, et al. The clinical application of ABCB1 genotyping in antidepressant treatment: a pilot study. CNS Spectr. 2014;19:165-75.

13. Breitfeld J, Scholl C, Steffens M, et al. Gene expression and proliferation biomarkers for antidepressant treatment resistance. Transl Psychiatry. 2017;7:e1061.

14. Cade JF. Lithium salts in the treatment of psychotic excitement. Med J Aust. 1949;2:349-52.

15. Chekroud AM, Zotti RJ, Shehzad Z, et al. Cross-trial prediction of treatment outcome in depression: a machine learning approach. The Lancet Psychiatry. 2016;3:243-50.

16. Chen CH, Lee CS, Lee MT, et al. Variant GADL1 and response to lithium therapy in bipolar I disorder. N Engl J Med. 2014;370: 119-28.

17. Civelek M, Lusis AJ. Systems genetics approaches to understand complex traits. Nat Rev Genet. 2014;15:34-48.

18. Clark SL, Adkins DE, Aberg K, et al. Pharmacogenomic study of side-effects for antidepressant treatment options in STAR*D. Psychol Med. 2012;42:1151-62.

19. Clayton AH, Croft HA, Horrigan JP, et al. Bupropion extended release compared with escitalopram: effects on sexual functioning and antidepressant efficacy in 2 randomized, double-blind, placebo-controlled studies. J Clin Psychiatry. 2006;67:736-46.

20. Consortium C. Sparse whole-genome sequencing identifies two loci for major depressive disorder. Nature. 2015;523:588.

21. Cross-Disorder Group of the Psychiatric Genomics C. Genetic relationship between five psychiatric disorders estimated from genome-wide SNPs. Nat Genet. 2013;45:984-94.

22. Cross-Disorder Group of the Psychiatric Genomics C. Identification of risk loci with shared effects on five major psychiatric disorders: a genome-wide analysis. Lancet. 2013;381: 1371-9.

23. Deans E. Microbiome and mental health in the modern environment. J Physiol Anthropol. 2017;36:1.

24. Direk N, Williams S, Smith JA, et al. An analysis of two genomewide association meta-analyses identifies a new locus for broad depression phenotype. Biol Psychiatry. 2017;82:322-29.

25. Doherty JL, Owen MJ. Genomic insights into the overlap between psychiatric disorders: implications for research and clinical practice. Genome Med. 2014;6:29-9.

26. Domschke K, Dannlowski U, Ohrmann P, et al. Cannabinoid receptor 1 (CNR1) gene: impact on antidepressant treatment response and emotion processing in major depression. Eur Neuropsychopharmacol. 2008;18:751-9.

27. Domschke K, Hohoff C, Mortensen LS, et al. Monoamine oxidase a variant influences antidepressant treatment response in female patients with major depression. Prog Neuropsychopharmacol Biol Psychiatry. 2008;32:224-8.

28. Domschke K, Tidow N, Schwarte K, et al. Serotonin transporter gene hypomethylation predicts impaired antidepressant treatment response. Int J Neuropsychopharmacol. 2014;17:1167-76.

29. Dusi N, Barlati S, Vita A, et al. Brain structural effects of antidepressant treatment in major depression. Curr Neuropharmacol. 2015;13:458-65. 
30. Fabbri C, Di Girolamo G, Serretti A. Pharmacogenetics of antidepressant drugs: an update after almost 20 years of research. Am J Med Genet B Neuropsychiatr Genet. 2013;162B:487-520.

31. Fabbri C, Serretti A. Pharmacogenetics of major depressive disorder: top genes and pathways toward clinical applications. Curr Psychiatry Rep. 2015;17:50.

32. Fava M, Rush AJ, Trivedi MH, et al. Background and rationale for the sequenced treatment alternatives to relieve depression (STAR*D) study. Psychiatr Clin North Am. 2003;26:457-494, x.

33. Ferrari AJ, Charlson FJ, Norman RE, et al. Burden of depressive disorders by country, sex, age, and year: findings from the global burden of disease study 2010. PLoS Med. 2013;10:e1001547.

34. Ferrari AJ, Stockings E, Khoo JP, et al. The prevalence and burden of bipolar disorder: findings from the Global Burden of Disease Study 2013. Bipolar Disord. 2016;18:440-50.

35. Flint J, Kendler KS. The genetics of major depression. Neuron. 2014;81:484-503.

36. Freund CL, Clayton EW, Wilfond BS. Natural settings trialsimproving the introduction of clinical genetic tests. J Law, Med Ethics: J Am Soc Law, Med Ethics. 2004;32:106-10.

37. Garcia-Gonzalez J, Tansey KE, Hauser J, et al. Pharmacogenetics of antidepressant response: a polygenic approach. Prog NeuroPsychopharmacol Biol Psychiatry. 2017;75:128-34.

38. Garriock HA, Kraft JB, Shyn SI, et al. A genomewide association study of citalopram response in major depressive disorder. Biol Psychiatry. 2010;67:133-8.

39. Geddes JR, Burgess S, Hawton K, et al. Long-term lithium therapy for bipolar disorder: systematic review and meta-analysis of randomized controlled trials. Am J Psychiatry. 2004;161:217-22.

40. Geoffroy PA, Curis E, Courtin C, et al. Lithium response in bipolar disorders and core clock genes expression. World J Biol Psychiatry. 2017:1-14.

41. Global Burden of Disease Study C. Global, regional, and national incidence, prevalence, and years lived with disability for 301 acute and chronic diseases and injuries in 188 countries, 1990-2013: a systematic analysis for the Global Burden of Disease study 2013. Lancet. 2015;386:743-800.

42. Golubnitschaja O, Baban B, Boniolo G, et al. Medicine in the early twenty-first century: paradigm and anticipation -EPMA position paper 2016. EPMA J. 2016;7:23.

43. Golubnitschaja O, Costigliola V, EPMA. EPMA summit 2014 under the auspices of the presidency of Italy in the EU: professional statements. EPMA J. 2015;6:4

44. Golubnitschaja O, Costigliola V, EPMA. General report \& recommendations in predictive, preventive and personalised medicine 2012: white paper of the European Association for Predictive, Preventive and Personalised Medicine. EPMA J. 2012;3:14.

45. Golubnitschaja O, Kinkorova J, Costigliola V. Predictive, preventive and personalised medicine as the hardcore of 'horizon 2020': EPMA position paper. EPMA J. 2014;5:6.

46. Gratten J, Wray NR, Keller MC, et al. Large-scale genomics unveils the genetic architecture of psychiatric disorders. Nat Neurosci. 2014;17:782-90.

47. Grof P, Duffy A, Cavazzoni P, et al. Is response to prophylactic lithium a familial trait? J Clin Psychiatry. 2002;63:942-7.

48. Guilloux JP, Bassi S, Ding Y, et al. Testing the predictive value of peripheral gene expression for nonremission following citalopram treatment for major depression. Neuropsychopharmacology. 2015;40:701-10.

49. Halushka MK, Fan JB, Bentley K, et al. Patterns of singlenucleotide polymorphisms in candidate genes for blood-pressure homeostasis. Nat Genet. 1999;22:239-47.

50. Hennings JM, Owashi T, Binder EB, et al. Clinical characteristics and treatment outcome in a representative sample of depressed inpatients - findings from the Munich Antidepressant Response Signature (MARS) project. J Psychiatr Res. 2009;43:215-29.
51. Hieronymus F, Emilsson JF, Nilsson S, et al. Consistent superiority of selective serotonin reuptake inhibitors over placebo in reducing depressed mood in patients with major depression. Mol Psychiatry. 2016;21:523-30.

52. Hirschfeld RM. Efficacy of SSRIs and newer antidepressants in severe depression: comparison with TCAs. J Clin Psychiatry. 1999;60:326-35.

53. Hodgson K, Mufti SJ, Uher R, et al. Genome-wide approaches to antidepressant treatment: working towards understanding and predicting response. Genome Med. 2012;4:52.

54. Horstmann S, Lucae S, Menke A, et al. Polymorphisms in GRIK4, HTR2A, and FKBP5 show interactive effects in predicting remission to antidepressant treatment. Neuropsychopharmacology. 2010;35:727-40.

55. Hou L, Bergen SE, Akula N, et al. Genome-wide association study of 40,000 individuals identifies two novel loci associated with bipolar disorder. Hum Mol Genet. 2016;25:3383-94.

56. Hou L, Heilbronner U, Degenhardt F, et al. Genetic variants associated with response to lithium treatment in bipolar disorder: a genome-wide association study. Lancet. 2016;387:1085-93.

57. Hughes B. Novel consortium to address shortfall in innovative medicines for psychiatric disorders. Nat Rev Drug Discov. 2009;8:523-4.

58. Hunter AM, Leuchter AF, Power RA, et al. A genome-wide association study of a sustained pattern of antidepressant response. $\mathrm{J}$ Psychiatr Res. 2013;47:1157-65.

59. Hyde CL, Nagle MW, Tian C, et al. Identification of 15 genetic loci associated with risk of major depression in individuals of European descent. Nat Genet. 2016;48:1031-6.

60. Iniesta R, Malki K, Maier W, et al. Combining clinical variables to optimize prediction of antidepressant treatment outcomes. J Psychiatr Res. 2016;78:94-102.

61. Iniesta R, Stahl D, Mcguffin P. Machine learning, statistical learning and the future of biological research in psychiatry. Psychol Med. 2016;46:2455-65.

62. Ising M, Lucae S, Binder EB, et al. A genome-wide association study points to multiple loci predicting antidepressant treatment outcome in depression. Arch Gen Psychiatry. 2009;66:966-75.

63. Kauzna-Czapliska J, Zurawicz E, Michalska M, et al. A focus on homocysteine in autism. Acta Biochim Pol. 2013;60:137-42.

64. Kawaguchi DM, Glatt SJ. GRIK4 polymorphism and its association with antidepressant response in depressed patients: a metaanalysis. Pharmacogenomics. 2014;15:1451-9.

65. Kinkorová J. Biobanks in the era of personalized medicine: objectives, challenges, and innovation. EPMA J. 2016;7:4.

66. Kleindienst N, Engel RR, Greil W. Psychosocial and demographic factors associated with response to prophylactic lithium: a systematic review for bipolar disorders. Psychol Med. 2005;35:1685-94.

67. Korgaonkar M, Goldstein-Piekarski A, Fornito A, et al. 253. Functional connectome networks underlying outcomes of antidepressant medication in major depressive disorders. Biol Psychiatry. 2017;81:S104.

68. Lee MS, Lee HY, Lee HJ, et al. Serotonin transporter promoter gene polymorphism and long-term outcome of antidepressant treatment. Psychiatr Genet. 2004;14:111-5.

69. Lemke HU, Golubnitschaja O. Towards personal health care with model-guided medicine: long-term PPPM-related strategies and realisation opportunities within 'horizon 2020'. EPMA J. 2014;5:8.

70. Li X, Bijur GN, Jope RS. Glycogen synthase kinase-3beta, mood stabilizers, and neuroprotection. Bipolar Disord. 2002;4:137-44.

71. Lichtenstein P, Yip BH, Björk C, et al. Common genetic determinants of schizophrenia and bipolar disorder in Swedish families: a population-based study. Lancet. 2009;373:234-9.

72. Malki K, Tosto MG, Mouriño-Talín H, et al. Highly polygenic architecture of antidepressant treatment response: comparative 
analysis of SSRI and NRI treatment in an animal model of depression. Am J Med Genet B Neuropsychiatr Genet. 2017;174:23550.

73. Mamdani F, Alda M, Grof P, et al. Lithium response and genetic variation in the CREB family of genes. American journal of medical genetics. B, Neuropsychiatr Genet: Publi Int Soc Psychiatr Genet. 2008;147B:500-4.

74. Masui T, Hashimoto R, Kusumi I, et al. A possible association between the $-116 \mathrm{C} / \mathrm{G}$ single nucleotide polymorphism of the $\mathrm{XBP} 1$ gene and lithium prophylaxis in bipolar disorder. Int J Neuropsychopharmacol. 2006;9:83-8.

75. Masui T, Hashimoto R, Kusumi I, et al. A possible association between missense polymorphism of the breakpoint cluster region gene and lithium prophylaxis in bipolar disorder. Prog NeuroPsychopharmacol Biol Psychiatry. 2008;32:204-8.

76. Matsumoto Y, Fabbri C, Pellegrini S, et al. Serotonin transporter gene: a new polymorphism may affect response to antidepressant treatments in major depressive disorder. Mol Diagn Ther. 2014;18: 567-77.

77. Mcguffin P, Rijsdijk F, Andrew M, et al. The heritability of bipolar affective disorder and the genetic relationship to unipolar depression. Arch Gen Psychiatry. 2003;60:497-502.

78. Mcmahon FJ, Buervenich S, Charney D, et al. Variation in the gene encoding the serotonin $2 \mathrm{~A}$ receptor is associated with outcome of antidepressant treatment. Am J Hum Genet. 2006;78: $804-14$

79. Menke A, Binder EB. Epigenetic alterations in depression and antidepressant treatment. Dialogues Clin Neurosci. 2014;16: 395-404.

80. Merikangas KR, Jin R, He J-P, et al. Prevalence and correlates of bipolar spectrum disorder in the world mental health survey initiative. Arch Gen Psychiatry. 2011;68:241-51.

81. Min W, Li T, Ma X, et al. Monoamine transporter gene polymorphisms affect susceptibility to depression and predict antidepressant response. Psychopharmacology. 2009;205:409-17.

82. Mrazek DA. Psychiatric pharmacogenomic testing in clinical practice. Dialogues Clin Neurosci. 2010;12:69-76.

83. Mrazek DA, Biernacka JM, Mcalpine DE, et al. Treatment outcomes of depression: the pharmacogenomic research network antidepressant medication pharmacogenomic study. J Clin Psychopharmacol. 2014;34:313-7.

84. Mühleisen TW, Leber M, Schulze TG, et al. Genome-wide association study reveals two new risk loci for bipolar disorder. Nat Commun. 2014;5:3339.

85. Murray CJL, Barber RM, Foreman KJ, et al. Global, regional, and national disability-adjusted life years (DALYs) for 306 diseases and injuries and healthy life expectancy (HALE) for 188 countries, 1990-2013: quantifying the epidemiological transition. Lancet. 2015;386:2145-91.

86. Niitsu T, Fabbri C, Bentini F, et al. Pharmacogenetics in major depression: a comprehensive meta-analysis. Prog NeuroPsychopharmacol Biol Psychiatry. 2013;45:183-94.

87. O'donovan MC, Owen MJ. The implications of the shared genetics of psychiatric disorders. Nat Med. 2016;22:1214-9.

88. Oestergaard S, Moldrup C. Anticipated outcomes from introduction of 5-HTTLPR genotyping for depressed patients: an expert Delphi analysis. Public Health Genomics. 2010;13:406-14.

89. Papakostas GI, Fava M, Thase ME. Treatment of SSRI-resistant depression: a meta-analysis comparing within- versus across-class switches. Biol Psychiatry. 2008;63:699-704.

90. Perlis RH. Cytochrome P450 genotyping and antidepressants. BMJ: Br Med J. 2007;334:759-9.

91. Peters EJ, Slager SL, Mcgrath PJ, et al. Investigation of serotoninrelated genes in antidepressant response. Mol Psychiatry. 2004;9: $879-89$.
92. Psychiatric GCBDWG. Large-scale genome-wide association analysis of bipolar disorder identifies a new susceptibility locus near ODZ4. Nat Genet. 2011;43:977-83.

93. Purcell SM, Wray NR, Stone JL, et al. Common polygenic variation contributes to risk of schizophrenia and bipolar disorder. Nature. 2009;460:748-52.

94. Ritchie MD, Holzinger ER, Li R, et al. Methods of integrating data to uncover genotype-phenotype interactions. Nat Rev Genet. 2015;16:85-97.

95. Rush AJ, Fava M, Wisniewski SR, et al. Sequenced treatment alternatives to relieve depression $(\mathrm{STAR} * \mathrm{D})$ : rationale and design. Control Clin Trials. 2004;25:119-42.

96. Rybakowski JK, Dmitrzak-Weglar M, Kliwicki S, et al. Polymorphism of circadian clock genes and prophylactic lithium response. Bipolar Disord. 2014;16:151-8.

97. Rybakowski JK, Dmitrzak-Weglarz M, Suwalska A, et al. Dopamine D1 receptor gene polymorphism is associated with prophylactic lithium response in bipolar disorder. Pharmacopsychiatry. 2009;42:20-2.

98. Rybakowski JK, Suwalska A, Skibinska M, et al. Prophylactic lithium response and polymorphism of the brain-derived neurotrophic factor gene. Pharmacopsychiatry. 2005;38:166-70.

99. Schulze TG, Alda M, Adli M, et al. The international consortium on lithium genetics (ConLiGen): an initiative by the NIMH and IGSLI to study the genetic basis of response to lithium treatment. Neuropsychobiology. 2010;62:72-8.

100. Serretti A, Lilli R, Lorenzi C, et al. Tryptophan hydroxylase gene and response to lithium prophylaxis in mood disorders. J Psychiatr Res. 1999;33:371-7.

101. Serretti A, Lilli R, Mandelli L, et al. Serotonin transporter gene associated with lithium prophylaxis in mood disorders. Pharmacogenomics J. 2001;1:71-7.

102. Serretti A, Malitas PN, Mandelli L, et al. Further evidence for a possible association between serotonin transporter gene and lithium prophylaxis in mood disorders. Pharmacogenomics J. 2004;4: 267-73.

103. Silberberg G, Levit A, Collier D, et al. Stargazin involvement with bipolar disorder and response to lithium treatment. Pharmacogenet Genomics. 2008;18:403-12.

104. Singh AB, Baune BT, Hamilton A, et al. Psychotropic pharmacogenetics - distraction or destiny? Aust N Z J Psychiatry. 2017;51: 665-7.

105. Soares JC, Gershon S. The lithium ion: a foundation for psychopharmacological specificity. Neuropsychopharmacology. 1998;19:167-82

106. Song J, Bergen SE, Di Florio A, et al. Genome-wide association study identifies SESTD1 as a novel risk gene for lithiumresponsive bipolar disorder. Mol Psychiatry. 2016;21:1290-7.

107. Spencer CCA, Su Z, Donnelly P, et al. Designing genome-wide association studies: sample size, power, imputation, and the choice of genotyping Chip. PLoS Genet. 2009;5:e1000477.

108. Steen VM, Lovlie R, Osher Y, et al. The polymorphic inositol polyphosphate 1-phosphatase gene as a candidate for pharmacogenetic prediction of lithium-responsive manic-depressive illness. Pharmacogenetics. 1998;8:259-68.

109. Sullivan PF, Neale MC, Kendler KS. Genetic epidemiology of major depression: review and meta-analysis. Am J Psychiatr. 2000;157:1552-62.

110. Szczepankiewicz A, Rybakowski JK, Suwalska A, et al. Glucocorticoid receptor polymorphism is associated with lithium response in bipolar patients. Neuro Endocrinol Lett. 2011;32: 545-51.

111. Szczepankiewicz A, Skibinska M, Suwalska A, et al. The association study of three FYN polymorphisms with prophylactic lithium response in bipolar patients. Hum Psychopharmacol. 2009;24: 287-91. 
112. Tansey KE, Guipponi M, Hu X, et al. Contribution of common genetic variants to antidepressant response. Biol Psychiatry. 2013;73:679-82.

113. Tansey KE, Guipponi M, Perroud N, et al. Genetic predictors of response to serotonergic and noradrenergic antidepressants in major depressive disorder: a genome-wide analysis of individuallevel data and a meta-analysis. PLoS Med. 2012;9:e1001326.

114. The Genomes Project C. A global reference for human genetic variation. Nature. 2015;526:68-74.

115. Thomas L, Mulligan J, Mason V, et al. GENetic and clinical predictors of treatment response in depression: the GenPod randomised trial protocol. Trials. 2008;9:29.

116. Uher R, Perroud N, Ng MY, et al. Genome-wide pharmacogenetics of antidepressant response in the GENDEP project. Am J Psychiatry. 2010;167

117. Uher R, Tansey KE, Henigsberg N, et al. Common genetic variation and antidepressant efficacy in major depressive disorder: a meta-analysis of three genome-wide pharmacogenetic studies. Am J Psychiatr. 2013;170:207-17.

118. Van Der Weide J, Hinrichs JWJ. The influence of cytochrome P450 pharmacogenetics on disposition of common antidepressant and antipsychotic medications. Clin Biochem Rev. 2006;27:1725.

119. Vialou V, Feng J, Robison AJ, et al. Epigenetic mechanisms of depression and antidepressants action. Annu Rev Pharmacol Toxicol. 2013;53:59-87.

120. Whiteford HA, Degenhardt L, Rehm J, et al. Global burden of disease attributable to mental and substance use disorders: findings from the global burden of disease study 2010. Lancet. 2013;382:1575-86.

121. Winner J, Allen JD, Anthony Altar C, et al. Psychiatric pharmacogenomics predicts health resource utilization of outpatients with anxiety and depression. Transl Psychiatry. 2013;3: e242. 